\title{
An investigation of an autonomic innervation of the vertebral artery using monoamine histofluorescence
}

\section{J.A. Mitchell}

Department of Zoology \& Physiology, University of Wyoming, Laramie, USA

.

(C)2004, European Journal of Histochemistry

Blood flow to the hindbrain, via the paired vertebral arteries, must be uncompromised for adequate neurological functioning of its vital centres. Therefore, it would seem unlikely that the intracranial vertebral artery would need to vasoconstrict, thus reducing its blood flow. In order to investigate the existence and location of a noradrenaline-mediated constrictor mechanism in the wall of the intracranial vertebral artery, transverse sections of ten baboon and ten monkey vessels were stained with sucrose-potassium phosphate-glyoxylic acid (counterstained with malachite-green). This method allows the visualisation of catecholaminergic nerves when the sections are exposed to ultraviolet light. In this study of primate vascular tissue, however, none of the monkey or baboon vertebral artery sections showed the presence of noradrenergic nerves in the tunica media - tunica adventitia junction or penetrating the tunica media of the arteries. These findings indicate that the intracranial vertebral artery does not have a neurogenic vasomotor function in primates.

Note. This work was carried out in the School of Anatomical Sciences, University of the Witwatersrand, Johannesburg, South Africa.

Correspondence: Dr. Jeanette Mitchell, Department of Zoology \& Physiology, University of Wyoming, Laramie, Wyoming 82071-3166, USA. E-mail: mitchja@uwyo.edu

Paper accepted on September 26, 2003

European Journal of Histochemistry 2004; vol. 48 issue 2 [Apr-Jun]: 115-120
V ascular smooth muscle cells in cranial vessels react to various metabolic, neural and humoral stimuli (Purves, 1972; Ganong, 1997). For example, vasoconstriction results from stimulation of the vascular smooth muscle cells by noradrenaline, either secreted from sympathetic nerve varicosities and terminals, or circulating in the blood stream (Ganong, 1997). Furthermore, it is known that stretch of a vessel, such as that which may occur in the vertebral artery on end-of-range rotation of the cervical vertebral column (Rossitti and Volkmann, 1995; Kuether et al., 1997; Haynes and Milne, 2001), causes a calcium influx-related myogenic spasm (Davis et al., 1992a, 1992b). Vasoconstriction will decrease the vertebral artery diameter (thus, cross-sectional area) and reduce blood flow in the vessel (Bernoulli's principle: Ganong, 1997). Because the paired vertebral arteries supply the entire hindbrain (Williams et al., 1989) with essential oxygen and glucose, any change in blood flow because of vasoconstriction, if prolonged (when associated with stretch-induced myogenic vasospasm, for example), may influence neurological function. Therefore, it is of value to establish whether vasoconstriction in the vertebral artery is neurogenic, that is whether there is evidence for noradrenergic innervation of the artery, which may enhance vasoconstriction of myogenic origin and exacerbate the compromising effect on blood flow.

Since the introduction in the early 1960s of the Falck-Hillarp method to visualise catecholamines (such as noradrenaline) by formaldehyde-induced fluorescence, modified and improved techniques have been used routinely (Lindvall and Bjorklund, 1974; de la Torre and Surgeon, 1976; Guidry, 1999). Glyoxylic acid has replaced formaldehyde, as it was found to have a higher capacity to form strongly fluorescent fluorophores with dopamine and noradrenaline particularly (Lindvall et al., 1973; Bjorklund and Hokfelt, 1983). The bright 
green fluorescence seen (with maximum emission in the $470 \mathrm{~nm}$ wavelength) is a result of the formation of isoquinoline derivatives (fluorophores) from the catecholamines when exposed to glyoxylic acid (Lindvall and Bjorklund, 1974; Bjorklund and Hokfelt, 1983). The addition of sucrose and potassium phosphate to the glyoxylic acid enabled improved visualisation of finer structures such as preterminal and axonal varicosities in catecholaminergic nerves (de la Torre and Surgeon, 1976; Cottle et al., 1985).

This sucrose-potassium phosphate-glyoxylic acid (SPG) method has been shown to be superior, with increased specificity, sensitivity, and stability of results, and rapidity and simplicity of application (de la Torre and Surgeon, 1976). The technique has been used to visualise central and peripheral noradrenaline- and dopamine-containing neurones in a variety of tissues (brain, iris, heart, lung, kidney, adrenal gland and brown adipose tissue), in several different animals (rat, mole-rat, dog, rabbit and monkey) (Lindvall et al., 1973; Lindvall and Bjorklund, 1974; de la Torre and Surgeon, 1976; Cottle et al., 1985; Daly et al., 1997; Guidry, 1999).

The further improvement to the technique was reported by Guidry (1999) who counterstained the tissue with the stain malachite-green. This dye stained the non-catecholaminergic tissues an orange-red colour, the overall effect being to reduce the intrinsic, nonspecific, green autofluorescence of the tissues, enabling the catecholaminergic structures to appear to fluoresce more brightly against the darker background.

Despite these developments in catecholamine histofluorescence, there have been few investigations of the sympathetic innervation of cerebral blood vessels in the higher primates. There are no reports in the current literature of studies of the vertebral artery in this respect. However, in a pilot study using high performance liquid chromatography and electrochemical detection, noradrenaline was found in homogenized intracranial vertebral artery tissue in four baboons (Mitchell, 2002). These findings suggest that the smooth muscle wall of the vertebral artery has a sympathetic innervation and, thus, a vasomotor function. However, the noradrenaline-mediated constrictor mechanisms can only be activated in the vascular smooth muscle if noradrenergic nerves are present in the perivascular adventitia and have their nerve termi- nals close to the smooth muscle cells of the tunica media (Fawcett, 1994). This suggests that an attempt to demonstrate whether such nerves are sufficiently close to the smooth muscle cells of the artery wall to have a vasomotor function is indicated. Therefore, it was the purpose of this study to examine the intracranial vertebral artery further in order to localise such innervation in smaller sections of the vessel, in primates.

\section{Materials and Methods}

\section{Specimens}

Fresh human material was not available. Therefore, the vertebral arteries studied were obtained from baboon and vervet monkeys, on the assumption that these animals are likely to be representative of primates generally, and their anatomy is comparable to that of humans (Tominaga et al., 1995).

The right intracranial (fourth) part of the vertebral artery from ten adult, male and female vervet monkeys (Cercopithecus aethiops) and ten adult, male and female Chacma baboons (Papio ursinus) formed the sample in this study. Tissue was salvaged immediately after the death of animals used by other researchers. Ethics approval was obtained from the Animal Ethics Committee, University of the Witwatersrand, Johannesburg. The entire brain was removed and the vertebral arteries carefully dissected from the base of the brain. A small block of the medulla oblongata was left in situ, to support each vertebral artery. As each sample of tissue was removed from the animal, it was quickly frozen in isopentane cooled in liquid nitrogen. The medulla oblongata, which is known to contain catecholaminergically innervated blood vessels (Purves, 1972; Ganong, 1997), and the medulla of a baboon adrenal gland, containing noradrenalin-secreting cells, were used as the positive controls. All samples were stored at $-70^{\circ} \mathrm{C}$ until further use.

\section{Sectioning and staining of specimens}

Sections of $10 \mu \mathrm{m}$ were cut from each frozen vertebral artery specimen using a Reichert-Jung Cryocut 1800 cryostat, at $-15^{\circ} \mathrm{C}$. The first two to three sections of each specimen were mounted onto clean glass slides and dried on a hotplate at $60^{\circ} \mathrm{C}$. Each section was then routinely stained with toluidine blue-pyronin $Y$ solution, and examined under the light microscope at magnifications of $40 \mathrm{X}$ and 
100X to establish the orientation of each vertebral artery. The tissue blocks were trimmed and $10 \mu \mathrm{m}$ transverse serial sections were cut. These were mounted onto clean glass slides and allowed to reach room temperature before staining.

A pilot study to determine the best staining times for vascular tissue, using the modified SPG method of de la Torre and Surgeon (1976), was carried out. All reagents used were of analytical grade and were obtained from Saarchem (Pty) Ltd., Johannesburg, South Africa; Merck (Pty) Ltd., Darmstadt, Germany; BDH Chemicals Ltd., Poole, England, and Sigma Chemical Co., St. Louis, MO, USA. Three sets of slides, with two to three serial transverse sections of a vertebral artery specimen per slide, were prepared. The sections on each set of slides were stained and incubated for different times and temperatures, as follows:

(i) stained with SPG for 5 seconds, incubated for 20 minutes at $60^{\circ} \mathrm{C}$;

(ii) stained with SPG for 10 seconds, incubated for $80^{\circ} \mathrm{C}$ for 10 minutes;

(iii) stained with SPG for 10 seconds, incubated for $80^{\circ} \mathrm{C}$ for 5 minutes.

On comparing the strength of the fluorescence demonstrated in the sections stained with these three methods, the third staining method gave the best results, and was used in this study.

A further two sets of slides, with approximately ten serial sections per slide, were prepared for each of the ten monkey and ten baboon vertebral arteries and the baboon adrenal gland specimen. Each slide of the first set was immersed into a freshly prepared SPG solution at room temperature for 10 seconds. Each slide of the second set was immersed first for 5 seconds at room temperature into the malachite green-SPG solution, to mask background autofluorescence by counterstaining non-catecholaminergic tissues (Guidry, 1999), and then for a further 5 seconds into the SPG solution. In each case, excess fluid was drained from the slide and the area around the specimen was wiped dry with a clean soft tissue. The slides were left to air-dry at room temperature for 5 to 10 minutes. Once completely dry, the sections were covered with a few drops of light, clear liquid paraffin (African Medicines (Pty) Ltd., Johannesburg, South Africa). Following this, the sections treated with the SPG solution only were incubated in an oven at $80^{\circ} \mathrm{C}$ for 5 minutes (according to method (iii) above). Those sections treated with the malachite green-SPG solution were incubated at $95^{\circ} \mathrm{C}$ for 2.5 minutes exactly (Guidry, 1999). All slides were coverslipped with liquid paraffin and kept in the dark at $4^{\circ} \mathrm{C}$.

\section{Fluorescence microscopic examination of sections}

Each section was viewed with a Nikon Optiphot II photomicroscope fitted with an Episcopic Fluorescence EFD-3 attachment, using excitation (450-490nm wavelength) and barrier (520nm wavelength) filters to focus the ultraviolet light onto the specimen and the emitted light back through the microscope. This enabled the visualisation of positive fluorescence as a bright green colour. Magnifications of 50X to $1000 \mathrm{X}$ were used to study the sections and photographs were taken of relevant areas.

\section{Results}

\section{Control tissue}

Cells of the adrenal medulla fluoresced brightly in those sections stained with SPG only (Figure 1.1). When the malachite-green counterstain was used, much of the background brightness seen in Figure 1.1 was masked, leaving only the adrenergic cells showing as bright green foci of light (Figure 1.2).

In sections of the medulla oblongata, bright green fluorescence was detected, particularly around the lumina of the intra-parenchymal blood vessels, indicating the presence of catecholaminergic nerves here (Figure 1.3). This positive fluorescence was still detected after the background autofluorescence of the neural tissue was masked by the malachite green counterstain (Figure 1.4).

\section{Vertebral artery tissue}

In all the sections of the intracranial vertebral artery stained with SPG only, the vessel wall was clearly seen against the dark background of the lumen and the area external to the vessel. The internal elastic lamina fluoresced very brightly in all sections (Figure 2.1 and 2.3). Short, bright green lines of fluorescence were evident in the tunica media and adventitia of some of the arteries (Figure 2.1 and 2.3). Counterstaining with malachite-green solution reduced the autofluorescence of the elastic tissue of the internal elastic laminae and of the fluorescence, believed to be elastic fibres, in the tunica media and adjacent tunica adventitia of the vertebral arteries (Figure 2.2 and 2.4). There 

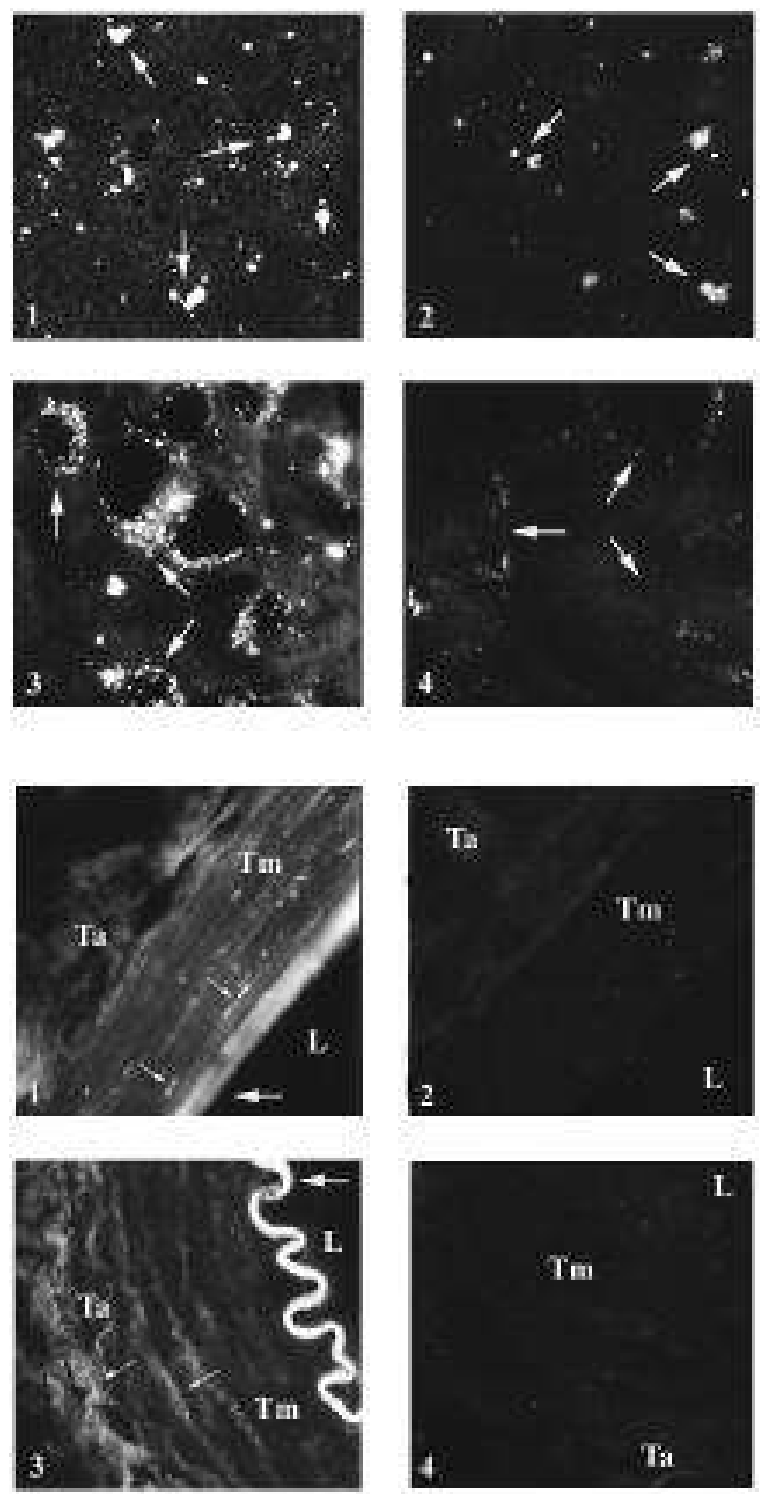

was no clear evidence of fluorescence in the region of the tunica media-tunica adventitia junction in any of the intracranial vertebral artery sections in any of the ten monkey or ten baboon specimens used in this study.

\section{Discussion and Conclusions}

In this study, the bright fluorescence shown in the control tissues (adrenal medulla and medulla oblongata, known to have catecholamine-containing cells and nerves, respectively) validated this SPG technique as a method for visualising catecholaminergic structures, thus supporting the reports of de la Torre and Surgeon (1976) and Daly et al. (1997). The absence of catecholaminer-
Figure 1. Visualisation of catecholamines using the SPG histofluorescence method (positive control). 1.1. A transverse section through the adrenal medulla of the baboon, stained with SPG. Brightly fluorescing catecholamine-containing cells (arrows) are seen against the dark background of the medulla of the gland. Magnification: 300X. 1.2. An adjacent section of the adrenal medulla of the baboon, stained with SPG and malachite-green. Only the bright fluorescence of the catecholaminergic cells (arrows) is clearly evident. Magnification: 300X.1.3. A section through the medulla oblongata of the monkey stained with SPG. Bright spots of fluorescence (arrows) are seen surrounding intraparenchymal capillaries. Magnification: 1200X. 1.4. An adjacent section of the medulla oblongata, treated with SPG and malachite-green. The perivascular catecholaminergic fluorescence (arrows) only is visualized. Magnification: 1200X.

Figure 2. Visualisation of catecholamines in the vertebral artery using the SPG histofluorescence method. 2.1. A transverse section through a segment of the vertebral artery (dilated) of the monkey stained with SPG. The internal elastic lamina fluoresces brightly (large arrow), and several foci of bright light are visible in the tunica adventitia and tunica media of the vessel (small arrows). Magnification: 1200X. 2.2. An adjacent section of the vertebral artery counterstained with malachite-green. The autofluorescence is masked and no brightly fluorescing structures are visible. Magnification: 1200X. 2.3. A segment of the vertebral artery (contracted) of the baboon in transverse section stained with SPG. Part of the brightly fluorescent internal elastic lamina (large arrow), and several bright, short, wavy lines (small arrows) in the tunicae media and adventitia of the artery are demonstrated. Magnification: 600X. 2.4. An adjacent section of the vertebral artery of the baboon treated with SPG and malachite-green. No bright fluorescence is visualised. Magnification: 600X. Keys: L = lumen; Ta = tunica adventitia; $\mathrm{Tm}$ = tunica media.

gic fluorescence in the tunica adventitia of the vertebral artery (after treatment with malachite green-SPG) was surprising, however, as noradrenergic innervation of this vessel was expected following the demonstration of noradrenaline in baboon tissue with HPLC (Mitchell, 2002). Nevertheless, it was appreciated that this chromatographic technique was able to verify the presence but not precise localization of the noradrenaline in the vascular tissue.

Assuming that the vertebral artery wall does contain noradrenergic nerves, there may be several reasons for the apparent absence of confirmatory fluorescence in the sections in the present study. First, the dark malachite-green stain may have masked the fluorescence of these fine nerves in the particu- 
lar specimens in this study. However, bright catecholaminergic fluorescence was demonstrated in the similarly stained control tissues (Figure 1.2 and 1.4). Therefore, the concentration of the malachitegreen stain used was considered to be adequate to mask autofluorescence but not catecholaminergically-derived fluorescence, although the sensitivity of the SPG-malachite green method in demonstrating very fine nerves remains in question.

Another explanation may be that the perivascular nerve plexus lies more in the external part of the tunica adventitia of the vertebral artery, with little penetration to the tunica media. It is possible that much of the tunica adventita was removed during the processing of the tissue in this fluorescence study of transverse sections of the vessel. As a result, nerves normally found in the outer part of this layer would not be visualised in the sections examined. However, in order to innervate the smooth muscle cells of an artery wall, the nerves would be expected to penetrate to, and thus be visualized in the tunica adventitia-tunica media junctional area. Such an arrangement of nerves was not seen in any of the 200 vertebral artery sections examined in the present study.

Lastly, if the density of the perivascular nerves in the vertebral artery is not high, it is possible that the particular sections studied were poorly innervated. Therefore, a lack of fluorescence in these sections should not be interpreted as a complete absence of these fine nerves in the vertebral artery. However, approximately $0.5 \mathrm{~mm}$ of each vertebral artery in each of the 10 monkeys and 10 baboons were sectioned, randomly along the length of the vessels, which was considered adequate to enable visualization of nerves if present.

of the similar histochemical studies reported in the literature, several authors have described perivascular nerve plexuses in the rat, although none of these authors discussed the exact localisation of these nerves in the wall of the vessel. Santer (1982), for example, showed perivascular nerve plexuses in the common and internal carotid arteries. Jones (1982) also visualised sympathetic nerve fibres in relation to major arteries at the base of the brain in the rat, and parenchymal arterioles for a short distance after branching from arteries.

Kobayashi et al. (1981) demonstrated aminergic nerves surrounding the proximal parts of the major cerebral arteries, and noted that this perivascular innervation was dense in the anterior but sparse in the posterior cerebral circulation in the rat brain. This would support the assumption of a lower density of these nerves in the wall of the baboon vertebral artery, which forms part of the posterior circulation of the brain. Furthermore, Saltzman et al. (1992) described an adrenergic nerve plexus situated close to the smooth muscle of arterioles in rat skeletal muscle, suggestive of neural control of vascular tone. Nevertheless, they do point out that there were substantial variations in the density of these nerves along the length of the arterioles, indicating that such control would be interspersed or minimal in the vessels. This view may be applied to the vertebral artery in the baboon to explain the apparent absence of noradrenergic nerves in the sections studied.

In addition, although de la Torre (1976) reported a positive result in the dog and the monkey, he was not able to demonstrate aminergic innervation of intracerebral arterioles in the rat brain, using the SPG method. This is, however, the only investigation of primate (monkey) tissue reported, and a similar positive result could be expected in baboon tissue. The only study which examined a possible noradrenergic innervation of the smooth muscle cells was reported by Baker and McDonald (1992). These authors were able to show, using the glyoxylic acid method, that catecholaminergic axons were associated with arterioles in the mucosa of the trachea of the rat. However, a direct innervation of the vascular smooth muscle cells was not demonstrated, nor were the nerves thought to supply the posterior, intercartilagenous, tracheal smooth muscle cells. Although these particular authors all used the glyoxylic acid method based on the original technique of Falck-Hillarp, none of them used a counter-staining technique, nor discussed the possibility of autofluorescence confounding their results.

These conflicting reports indicate that further research is necessary to try to establish the prevalence of a perivascular noradrenergic innervation in the cranial vessels, and in the vertebral artery in particular. The available evidence of noradrenergic nerves in some of these arteries does not demonstrate conclusively that they innervate the vascular smooth muscle cells. The paucity of such closelyrelated noradrenergic nerves in the vertebral artery, as suggested by the findings of the present study, indicates that the intracranial vertebral artery in primates does not have a neurogenic vasoconstrictor function. This would support the view that the 
vertebral artery is a distributing artery (Purves, 1972; Fawcett, 1994; Ganong, 1997) and, as such, functions to transport blood between the subclavian artery and the small parenchymal arterioles of the hindbrain. The latter vessels have been shown to be innervated by noradrenergic nerves and to play a role in cerebral perfusion through the autoregulation of blood flow (Purves, 1972; Ganong, 1997). Thus, more sensitive and specific tests to demonstrate direct innervation of vascular smooth muscle cells would be of value in providing unequivocal evidence for or against a neurogenic vasomotor function in the vertebral artery in primates.

\section{Acknowledgments}

This research was supported by a grant from the Medical Faculty Endowment Research Fund of the University of the Witwatersrand, Johannesburg, South Africa. The work was submitted by the author as part of the requirements for a PhD degree, awarded by the University of the Witwatersrand, Johannesburg, South Africa.

\section{References}

Baker DG, McDonald DM. Distribution of catecholamine-containing nerves on blood vessels of the rat trachea. J Comp Neurol 1992; 325:38-46.

Bjorklund A, Hokfelt T. Handbook of Chemical Neuroanatomy. Vol.1: Methods in Chemical Neuroanatomy. Elsevier, New York; 1983.

Cottle MKW, Cottle WH, Perusse F, Bukowiecki LJ. An improved glyoxylic acid technique for the histochemical localization of catecholamines in brown adipose tissue. Histochem J 1985;17:127988.

Daly TJM, Williams LA, Buffenstein R. Catecholaminergic innervation of interscapular brown adipose tissue in the naked mole-rat (Heterocephalus glaber). J Anat 1997;190:321-6.

Davis MJ, Donovitz JA, Hood JD. Stretch-activated single-channel and whole cell currents in vascular smooth muscle cells. Amer J Physiol 1992a; 262:C1083-C8.

Davis MJ, Meininger GA, Zawieja DC. Stretch-induced increases in intracellular calcium of isolated vascular smooth muscle cells. Amer J Physiol 1992b;236:H1292-H9.

de la Torre JC. Evidence for central innervation of intracranial blood vessels: local cerebral blood flow measurements and histofluores- cence analysis by the sucrose-phosphate-glyoxylic acid (SPG) method. Neuroscience 1976;1:455-7.

de la Torre JC, Surgeon JW. A methodological approach to rapid and sensitive monoamine histofluorescence using a modified glyoxylic acid technique: The SPG method. Histochem 1976; 49:81-93.

Fawcett D. Bloom and Fawcett: A Textbook of Histology. 12th ed. Chapman and Hall, New York; 1994.

Ganong WF. Review of medical physiology. $18^{\text {th }}$ edition. Appleton and Lange, editors. Stamford: Connecticut; USA. 1997.

Guidry G. A method for counterstaining tissues in conjunction with the glyoxylic acid condensation reaction for detection of biogenic amines. J Histochem Cytochem 1999;47:261-4.

Haynes M, Milne N. Color duplex sonographic findings in human vertebral arteries during cervical rotation. J Clin Ultrasound 2001; 29:14-24.

Jones BE. Relationship between catecholamine neurons and cerebral blood vessels studied by their simultaneous fluorescent revelation in the rat brainstem. Brain Res Bull 1982;9:33-44.

Kuether TA, Nesbit GM, Clark WM, Barnwell SL. Rotational vertebral artery occlusion: a mechanism of vertebrobasilar insufficiency. Neurosurg 1997;41:427-32.

Kobayashi S, Tsukahara S, Sugita K, Nagata T. Adrenergic and cholinergic innervation of rat cerebral arteries. Consecutive demonstration on whole mount preparations. Histochem 1981;70:129-38.

Lindvall 0, Bjorklund A. The glyoxylic acid fluorescence histochemical method: a detailed account of the methodology for the visualization of central catecholamine neurons. Histochem 1974;39:97-127.

Lindvall 0, Bjorklund A, Falck B. Glyoxylic acid condensation: A new fluorescence histochemical method for sensitive and detailed tracing of central catecholamine neurons. In Anonymous Frontiers in catecholamine research. Pergamon Press, London; 1973.

Mitchell, JA. 2002 The vertebral artery: An anatomical, histological and functional study. PhD thesis, University of the Witwatersrand, Johannesburg.

Purves M. The Physiology of the Cerebral Circulation. Cambridge University Press; Cambridge; 1972.

Rossitti S, Volkmann R. Changes of blood flow velocity indicating mechanical compression of the vertebral arteries during rotation of the head in the normal human measured with transcranial Doppler sonography. Arq Neuropsiquiatr 1995;53:26-33.

Saltzman D, DeLano FA, Schmid-Schonbein GW. The microvasculature in skeletal muscle. VI. Adrenergic innervation of arterioles in normatensive and spontaneously hypertensive rats. Microvasc Res 1992;44:263-73.

Santer RM. Fluorescence histochemical observations on the adrenergic innervation of the cardiovascular system in the aged rat. Brain Res Bull 1982;9:667-72.

Tominaga T, Dickman C, Sonntag V, Coons S. Comparative anatomy of the baboon and the human cervical spine. Spine 1995;20:131-7.

Williams PL, Warwick R, Dyson M, Bannister LH. Gray's anatomy. $37^{\text {th }}$ ed. Churchill Livingstone: Edinburgh; 1989. 